\title{
Cockayne syndrome type: a very rare association with hemorrhagic stroke
}

\author{
Başak Atalay $^{1 \oplus}$, Mine Sorkun $^{1 \oplus}$, Elif Yüksel Karatoprak ${ }^{2 \odot}$ \\ Departments of ${ }^{1}$ Radiology and ${ }^{2}$ Pediatric Neurology, İstanbul Medeniyet University Faculty of Medicine, Göztepe Training and \\ Research Hospital, Istanbul, Turkey.
}

\begin{abstract}
Background. Cockayne Syndrome (CS) is a rare autosomal recessive disorder that is mainly characterized by neurodevelopmental delay, cutaneous photosensitivity, and cachectic dwarfism. Genetic diagnosis is supported by the typical physical appearance and imaging findings of these patients.

Case. In our case, a 16-year-old female previously diagnosed as CS presented with right-sided hemiparesis. Magnetic Resonance Imaging (MRI) and Computed Tomography (CT) images showed diffuse cerebral and cerebellar atrophies, volume loss of brain stem, calcification of the bilateral basal ganglia, hemorrhage on the posterior limb of the left internal capsule, thalamus, and posterior periventricular area.
\end{abstract}

Conclusions. Cockayne syndrome is rarely associated with stroke; we report the clinical and neuroradiologic findings of CS presenting with a hemorrhagic stroke.

Key words: Cockayne syndrome, hemorrhagic stroke, magnetic resonance imaging, computed tomography.

Cockayne Syndrome (CS) is a rare autosomal recessive disorder with a prevalence of 2.5 per million. ${ }^{1}$ It was first described in 1936 by Edward Cockayne. ${ }^{2}$ Cockayne Syndrome is a multisystem disorder classified as a nucleotide excision repair disease characterized by neurodevelopmental delay, cutaneous photosensitivity, pigmentary retinopathy, neurosensory hearing loss, dental caries, cachectic dwarfism with senile-like appearance, dementia, endocrinopathies, progressive spasticity, ataxia, peripheral neuropathy, osteopenia, kyphosis, and joint contractures. ${ }^{3,4}$

Neurological manifestations are the main cause for morbidity of the disease. Herein we report a rare case of CS presenting with hemorrhagic stroke.

$凶$ Başak Atalay

basak_hosgoren@yahoo.com

Received 24th November 2020, revised 10th January 2021, accepted 14th February 2021.

\section{Case Report}

A 16 year old female was admitted to our hospital with right-sided hemiparesis for two weeks. She was diagnosed with CS at the age of ten years clinically and genetically (CS type A, Homozygous C.593-594dup frameshift pathogenic variation in ERCC8 gene). Her weight was $11 \mathrm{~kg}$, and her blood pressure was 90/60 mmHg. Examination revealed characteristic manifestations of CS such as cachectic dwarfism, progeroid face, bilateral cataracts, intellectual disability, microcephaly, joint contractures in the lower extremities, and dental caries (Fig. 1). Her history included full term, birth with 2500 gr weight, and normal developmental milestones until the age of one. Then, developmental delay and growth failure was detected. Her current examination revealed right sided hemiparesis with Medical Research Council Scale for muscle strength of 3/5 in the right upper and lower extremities and spasticity and contractures in the extremities. The patient had sensorineural hearing loss. Tests for lipid profile, renal and hepatic functions, serum 


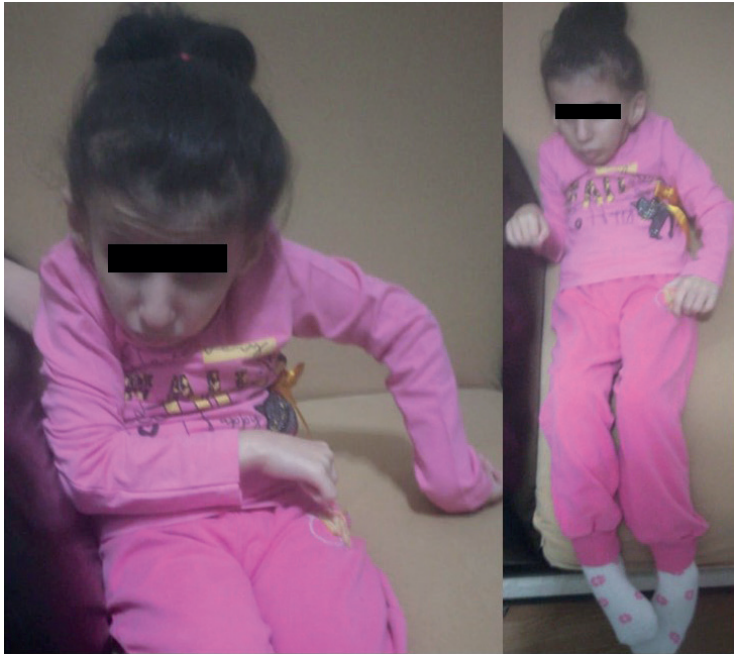

Fig. 1. Characteristic face, cachectic phenotype, joint contractures of the patient with Cockayne syndrome are shown.

homocysteine level, antithrombin III, proteins $\mathrm{C}$ and S, factor V Leiden, anticardiolipin antibodies, antiphospholipid antibodies, and antinuclear antibodies were normal.

Magnetic resonance imaging (MRI) showed diffuse cerebral and cerebellar atrophies with ventricular dilatation and widened sulci, volume loss of brain stem, and bilateral basal ganglia calcification. On T1 weighted images, there was high signal intensity on the posterior limb of the left internal capsule, thalamus, and posterior periventricular area suggesting hemorrhage. Restricted diffusion was seen on the posterior limb of the internal capsule and thalamus due to late subacute hemorrhage (Fig. 2).

\section{Subsequent computed tomography (CT)} revealed calcification in the bilateral basal ganglia and periventricular white matter of frontal and parietal lobes; CT also demonstrated hemorrhage on the posterior limb of the left internal capsule and thalamus (Fig. 3). No significant pathology was detected in CT and MR angiography studies regarding intracranial vascular structures. The patient previously diagnosed as CS, presented with hemorrhagic stroke during the course of the disease.
Permission was obtained from the parents and informed consent was obtained from the family.

\section{Discussion}

Cockayne Syndrome is a rare multisystem disorder characterized by a variety of clinical features, including severe neurological manifestations. Patchy and segmental demyelination, neuronal loss without major brain malformations, calcifications, vascular changes with accelerated atherosclerosis and arteriolosclerosis, severe cerebellar atrophy, and ventricular enlargement are the main neuropathologic findings that can clinically manifest as microcephaly, intellectual disability, tremors, ataxia, seizures, strokes and subdural hemorrhages. ${ }^{1}$

The diagnosis is based on characteristic phenotype, clinical features, radiological findings, and specific genetic tests for DNA analysis, which measure the recovery of RNA transcription after exposure to ultraviolet (UV) radiation. ${ }^{1,5}$ Neuroimaging findings, including calcifications mostly in the basal ganglia and cerebral cortex, cerebral and cerebellar atrophy with subsequent ventricular dilation, abnormal white matter signal intensity on MRI are the cardinal features suggesting the diagnosis of CS. The high signal intensity of the cerebral white matter on T2 weighted images demonstrates the tigroid patterns of demyelination, which represents sparing of the perivascular white matter. ${ }^{1,3}$ Our patient had neuroimaging findings compatible with CS.

ERCC6 (CSB) and ERCC8 (CSA) genes were identified as the two major genes responsible for CS. ${ }^{6}$ The disease is classified as classical form Type I, early-onset Type II, and late-onset Type III. Type II patients show symptoms in the congenital period, while Type III patients may be affected in late childhood or adulthood. Indeed CS patients demonstrate similar clinical and neuroradiological findings, but the onset and progression of the disease vary among subgroups. With a progressive manner of 

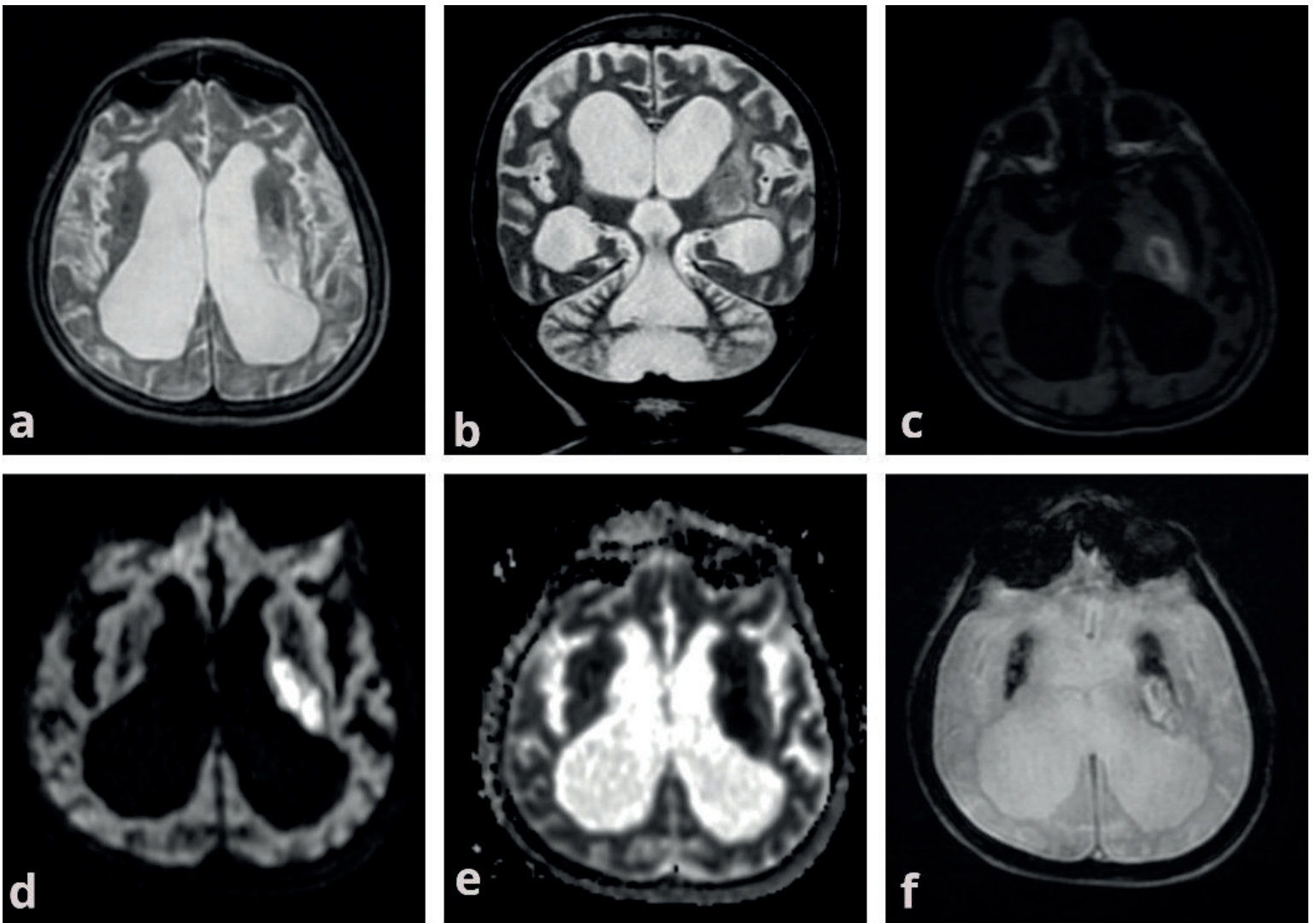

Fig. 2. Magnetic Resonance Imaging demonstrated diffuse cerebral and cerebellar atrophies with ventricular dilatation, and widened sulci, volume loss of brain stem on T2 weighted images (a,b). On T1 weighted images, there was high signal intensity on the posterior limb of the left internal capsule and thalamus which suggested subacute hemorrhage (c). Diffusion-weighted images revealed restricted diffusion on the posterior limb of the left internal capsule, thalamus, and posterior periventricular area with a decrease in the ADC value (d,e). Gradient echo imaging showed small areas of hypointensity in basal ganglia suggesting calcification (f).
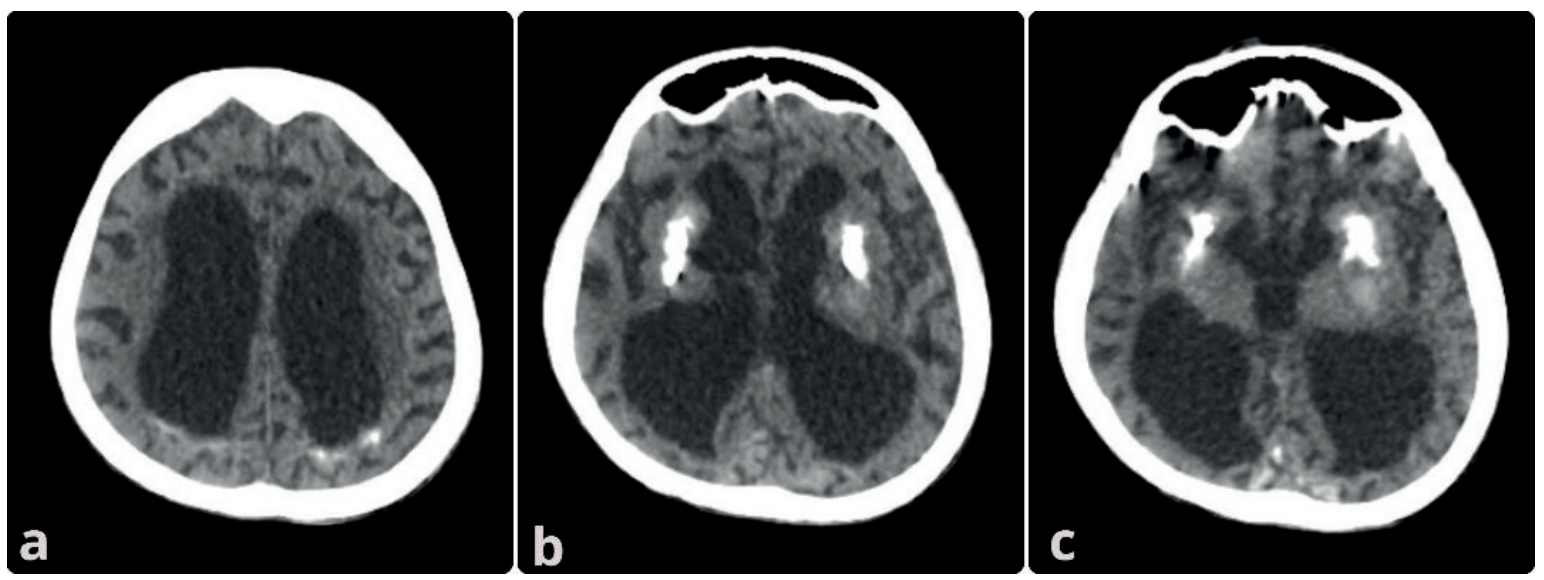

Fig. 3. Computed tomography (CT) revealed calcification in the bilateral basal ganglia and periventricular white matter of frontal and parietal lobes $(a, b, c)$. CT also demonstrated hemorrhage on the posterior limb of the left internal capsule and thalamus $(b, c)$. 
the disease, symptoms worsen with time. Development is very limited, especially in type II CS patients with a congenital form. Unlike Type II, in Type I classical form of the disease, development is normal in the first months of life, while motor delay begins at the end of the first year. Patients with CS Type III may have mild intellectual disability and learning difficulties. There is no threshold among the subtypes of the disease. Approximately $65 \%$ of patients show CSB gene mutation. Besides according to CSA and CSB linked mutations, there are no specific symptoms or severity of the disease. However, more severe forms (Type II) appear to be associated with CSB gene mutation, and milder forms (Type I) are associated with CSA gene mutation. ${ }^{7}$ Based on the genetic analysis (ERCC8 gene, CSA mutation) and clinical onset of the disease, our case was accepted as Type I, the classical form of CS. Solving the underlying molecular mechanisms with larger data will elucidate the CS genotypephenotypecorrelation.

The normal human aging process and its consequences are seen in the clinical features of CS. Premature onset of hypertension, microvascular pathology, which is characterized by string vessels, accelerated atherosclerosis, and arteriolosclerosis, are observed in the course of the disease and can lead to stroke. ${ }^{1,8,9}$ In our case, there were no radiological and laboratory findings suggesting cerebral atherosclerotic or thrombotic risk factors. Still, we havenot ruled out microvascular pathology since histopathological evaluation could not be performed. Literature on arterial disease in patients with CS is sparse, including ischemic or hemorrhagic strokes and transient ischemic attacks. $^{4,9-12}$ Mizuguchi and Itoh $^{12}$ declared a case of CS with hemorrhagic infarct in the parietal and insular cortex and demonstrated cerebrovascular changes histologically. The radiological findings of hemorrhagic stroke in CS had not been reported before. According to the literature, our case is the first to report the association between CS and hemorrhagic stroke radiologically.

In a recently published paper, Inceer et al. ${ }^{13}$ reported a Cockayne syndrome case as a rare cause of hemiplegia presented with subdural hematoma. Similar to this case report, bilateral subdural hematoma has been reported in another CS case with hemiplegia in the literature. ${ }^{14}$ Another CS case with hemiplegia, had normal brain imaging, while cerebral angiography showed stenotic plaques. $^{11}$ Although chronic subdural hematoma is mostly seen in elderly patients; case reports show that it can be seen with CS, which has premature aging signs. ${ }^{11,13}$ Various vascular changes may occur in patients with CS; pathological studies indicate that these patients have an increase in small arteries and arterioles in the subarachnoid space filled with non-atherosclerotic fibrotic tissue. The subarachnoid space and vascular structures increase with brain atrophy and subdural hemorrhages are related to enlarged subarachnoid spaces. ${ }^{8}$

In conclusion, it should be noted that besides the typical neurological features of CS, cerebral arterial disease may develop during the course of the disease. Although it is a rare association, hemorrhagic stroke may be accompanied by CS based on early atherosclerosis.

\section{Author contribution}

The authors confirm contribution to the paper as follows: study conception and design: BA, MS; data collection: BA, MS; analysis and interpretation of results: BA, MS, EYK; draft manuscript preparation: BA, MS, EYK. All authors reviewed the results and approved the final version of the manuscript.

\section{Conflict of interest}

The authors declare that they have no conflict of interest regarding the content of this article. 


\section{REFERENCES}

1. Karikkineth AC, Scheibye-Knudsen M, Fivenson E, Croteau DL, Bohr VA. Cockayne syndrome: clinical features, model systems and pathways. Ageing Res Rev 2017; 33: 3-17.

2. Cockayne EA. Dwarfism with retinal atrophy and deafness. Arch Dis Child 1936; 11: 1-8.

3. Koob M, Laugel V, Durand M, et al. Neuroimaging in Cockayne syndrome. AJNR Am J Neuroradiol 2010; 31: 1623-1630.

4. Rapin I, Weidenheim K, Lindenbaum Y, et al. Cockayne syndrome in adults: review with clinical and pathologic study of a new case. J Child Neurol 2006; 21: 991-1006.

5. Simon B, Oommen SP, Shah K, Mani SE, Gibikote S. Cockayne syndrome: characteristic neuroimaging features. Acta Neurol Belg 2015; 115: 427-428.

6. Henning, KA, Li L, Iyer $\mathrm{N}$, et al. The Cockayne syndrome group A gene encodes a WD repeat protein that interacts with CSB protein and a subunit of RNA polymerase II TFIIH. Cell 1995; 82: 555-564.

7. Laugel V. Cockayne syndrome: the expanding clinical and mutational spectrum. Mech Ageing Dev 2013; 134: 161-170.

8. Hayashi M, Miwa-Saito N, Tanuma N, Kubota M. Brain vascular changes in Cockayne syndrome. Neuropathology 2012; 32: 113-117.
9. Weidenheim KM, Dickson DW, Rapin I. Neuropathology of Cockayne syndrome: evidence for impaired development, premature aging, and neurodegeneration. Mech Ageing Dev 2009; 130: 619-636.

10. Crome L, Kanjilal GC. Cockayne's syndrome: case report. J Neurol Neurosurg Psychiatry 1971; 34: 171178.

11. Shirasaki N, Hayashi M, Handa Y, et al. Cockayne's syndrome presenting cerebral ischemic attack: case report. No To Shinkei 1986; 38: 871-875.

12. Mizuguchi M, Itoh M. A 35-year-old female with growth and developmental retardation, progressive ataxia, dementia and visual loss. Neuropathology 2005; 25: 103-106.

13. Inceer BS, Bulut O, Savaş Y. Cockayne syndrome as a rare cause of hemiplegia: review of the literature accompanied by a case report. Turk J Neurol 2019; 25: 36-38.

14. Shimoizumi H, Matsui M, Ito S, Miyao M, Kobayashi S. Cockayne syndrome complicated by acute subdural hemorrhage. Brain \& Development 1995; 17: 363-377. 